\title{
The energy dependence of relativistic nonradiative electron capture
}

\author{
J F McCann $\dagger$, J T Glassłand D S F Crothers $\ddagger$ \\ $\dagger$ Department of Physics, University of Durham, \\ Durham DH1 3LE, England. \\ $\ddagger$ The Theoretical and Computational Physics Research Division, \\ The Queen's University of Belfast, \\ Belfast BT7 1NN, Northern Ireland.
}

April 1996

\begin{abstract}
The energy dependence of the nonradiative electron capture crosssection is discussed in the relativistic domain. A simple analytic expression is obtained for inner-shell transitions using second-order perturbation theory. We have confirmed that the leading-order term is found to have the following energy dependence: $\sigma \sim E^{-1} \ln ^{2} E$. This is attributed to a combination of kinematic features of the process and retardation effects. Electron capture without change of spin is the dominant transition.
\end{abstract}

PACS 31.15.Md, 31.30.Jv, 34.70+e, 52.20.Hv

The asymptotic form of the electron capture process for relativistic energies has been a subject of debate for some time. An overview of this question has been given by Mukherjee et al (1992) and Bransden and McDowell (1993), while a detailed discussion is given by Eichler and Meyerhof (1995). In the relativistic domain, the physical constraints of energy and momentum matching conspire to make capture a weakly-coupled process in comparison to ionization or excitation. Indeed it is interesting to remark that for relativistic energies, and for small nuclear charges, vacuum coupling in the form 
of radiative electron capture is the dominant mechanism for charge exchange. As a consequence, the numerical results are primarily of academic interest in the extreme relativistic domain. Nonetheless, results for the nonradiative process ultimately provide important benchmarks and calibration standards for both experiment and theory (Eichler and Meyerhof 1995). Moreover the phenomenon has attracted attention in recent years not least because it is physically important for large nuclear charges, and this makes it accesssible to experimental studies up to and beyond the energy range $\mathrm{GeV} / \mathrm{u}$ (Anholt 1985). In the following discussion we use the Lorentz factor $\gamma$ as the energy parameter, and this is related to the laboratory frame kinetic energy through the usual relation $E=(\gamma-1) M_{P} c^{2}$, with $M_{P}$ the projectile mass.

Experimental data for relativistic collisions have been analysed and compared with theoretical models (Eichler 1990, Moiseiwitsch 1989, Deco and Rivarola 1987, Glass et al 1994), and satisfactory agreement has been obtained in many cases. However an intriguing question still remains open regarding the high-energy limit of the cross section, both in terms of the energy dependence and the size of the cross section. The uncertainty over this result reflects a well-known anomaly for high-energy nonrelativistic collisions, namely that electron capture is dominated by second-order terms. Nonetheless the dominance of the second-order term, via the Thomas mechanism, does not come into effect until the energies enter the relativistic domain. Paradoxically, as the energy rises further this mechanism becomes less important than the first-order process due to the contraction of suitable phase space. In fact it has been shown that the contribution to the cross section $(\sigma)$ given by the Thomas term decreases as $\sigma \sim \gamma^{-3}$ (Shakeshaft and Spruch 1979). This is much faster than the first-order term which behaves as $\sigma \sim \gamma^{-1}$ (Shakeshaft 1979) and ultimately it becomes negligible in the ultrarelativistic region.

In applying the Bates refinement of the ROBK1 approximation (Moiseiwitsch and Stockman 1980), the results were found to give rather good cross section estimates for the intermediate energy range. However this model also led to the prediction that $\sigma \sim \gamma^{-1}(\ln \gamma)^{2}$ at extremely high energies. The result that the nonorthogonal correction term eventually dominates the primary term (ROBK1) was suprising, and subsequently misgivings have been expressed regarding the validity of this approach (Eichler 1990). This result also contradicted other modified first-order models, such as relativistic B1B (Moiseiwitsch 1986) which predicted the result $\sigma \sim \gamma^{-1}$ (Toshima and Eich- 
ler 1990). Conversely it could be argued that the important influence of this correction term is not more suprising than the dominance of second-order terms, and reflects the weakness of the first-order process.

The picture in second-order theories is not so clear. Most of the work to date has centred on the well-known ROBK2 model (Moiseiwitsch 1985, Decker 1990) and relativistic impulse approximation RIA (Jakubassa-Amundsen and Amundsen 1980). Humphries and Moiseiwitsch (1985) followed the standard nonrelativistic approach to evaluating ROBK2 by using a linearisedpropagator peaking approximation as well as making expansions in $\alpha Z$ in the wave functions. This approximation reduces the $T$-matrix to a simple analytic form, and gives the result $\sigma \sim \gamma^{-1}$, as in ROBK1 though with a modified coefficient. Decker and Eichler (1993) managed to calculate the $T$ matrix exactly using numerical techniques, and in doing so queried the earlier ROBK 2 work on two counts. Firstly, when they compared their results with experimental data (which involves largish charges) they found that ROBK2 was in worse accord with experiment than ROBK1. This discrepancy has not as yet been fully accounted for in that the relative effects of the $\alpha Z$ expansions and the peaking approximation have not been isolated. Decker and Eichler (1993) also claimed signs of an energy dependence which contained logarithmic terms. Again possibly indicative of the size of the corresponding coefficient, the evidence was not conclusive even though they performed calculations for $\gamma>1000$.

Jakubassa-Amundsen and Amundsen (1980) in extending their work on the impulse approximation to the relativistic regime had argued that the ionization-like matrix element embedded in the RIA $T$-matrix would lead to logarithmic terms. The origin of these terms is the retardated potential. Indeed logarithmic correction terms are ubiquitous in high-energy atomic processes such as the stopping of relativistic charged particles. In both ROBK2 and RIA the possibility of retardation is obvious from the form of the $T$ matrix but as yet there has been no detailed mathematical study of both the origin of these terms as well their size in comparison to the ROBK1 amplitude. We have attempted to this effect. We will show that the multiplicative factor upon the logarithmic terms is of order $\alpha^{2}\left(Z_{P}+Z_{T}\right)^{2}$ compared with ROBK1. 


\section{First-order Approximations}

The cross section for electron capture $(\sigma)$, for a given impact energy in the laboratory frame, can be written in terms of the scattering amplitude $T(\boldsymbol{\eta})$ as follows (Glass et al 1996):

$$
\sigma(\gamma)=(2 \pi \gamma v)^{-2} \int d \boldsymbol{\eta}|T(\boldsymbol{\eta})|^{2}
$$

In this formula, $v$ is the relative speed of target $(T)$ and projectile $(P)$, and $\gamma=\left(1-v^{2} / c^{2}\right)^{-1 / 2}$. The momentum transfer variable is denoted by $\boldsymbol{\eta}$, and all quantities are expressed in atomic units $\left(|e|=m_{e}=\hbar=1\right.$, and $\left.\alpha=1 / c\right)$.

Specifically, we consider the capture of an electron from the target $1 \mathrm{~s}-$ state $\Phi_{T}$ to the projectile 1s-state $\left(\Phi_{P}\right)$. In this paper we use the following notation to describe these states:

$$
\Phi_{T}=\phi_{T}(\boldsymbol{r}) \mathrm{e}^{-i E_{T} t} \quad, \quad \Phi_{P}^{\prime}=\phi_{P}^{\prime}\left(\boldsymbol{r}^{\prime}\right) \mathrm{e}^{-i E_{P} t^{\prime}} .
$$

In the equation above, the functions are defined in the rest frames of the target and projectile nuclei respectively. The electron coordinate is denoted by $(\boldsymbol{r})$ with respect to the origin located at the target nucleus, and it is labelled $\left(\boldsymbol{r}^{\prime}\right)$ when referred to the projectile nucleus. These coordinates are related through the usual Lorentz transformation. All primed variables indicate that they are associated with the rest frame of the projectile nucleus. The atomic energies, which include the electron rest mass, are denoted by $E_{P, T}$. Then the relativistic first-order OBK (ROBK1) approximation takes the form (Shakeshaft 1979):

$$
T_{\mathrm{ROBK} 1}(\boldsymbol{\eta})=(2 \pi)^{3 / 2} \int d^{3} \boldsymbol{s} \tilde{\phi}_{P}^{\dagger \prime}\left(\boldsymbol{K}^{\prime}+\boldsymbol{s}\right) \tilde{V}_{P}^{\prime}(\boldsymbol{s}) S \tilde{\phi}_{T}(\boldsymbol{K}) .
$$

The Lorentz transform operator for a boost from the target to the projectile frame is denoted by $S$. The tilde signifies the Fourier transform through the definition

$$
\tilde{f}(\boldsymbol{p})=(2 \pi)^{-3 / 2} \int d^{3} \boldsymbol{r} \mathrm{e}^{-1} \boldsymbol{p} \cdot \boldsymbol{r} f(\boldsymbol{r}) .
$$

The momentum transfer vectors are simply given by:

$$
\begin{aligned}
& \boldsymbol{K}=\boldsymbol{K}_{\perp}+\boldsymbol{K}_{\|}=\boldsymbol{\eta}+\hat{\boldsymbol{v}}\left(\gamma E_{T}-E_{P}\right) /(\gamma v), \\
& \boldsymbol{K}^{\prime}=\boldsymbol{K}_{\perp}^{\prime}+\boldsymbol{K}_{\|}^{\prime}=\boldsymbol{\eta}-\hat{\boldsymbol{v}}\left(\gamma E_{P}-E_{T}\right) /(\gamma v) .
\end{aligned}
$$


Evidently at very high velocities $(\gamma \rightarrow \infty)$ the only energy dependence in the expression for $T_{\text {ROBK1 }}$ is due to the operator $S$, and thus $T \sim \gamma^{1 / 2}$. Then it clear that in the same limit $\sigma \sim \gamma^{-1}$. This energy dependence is a feature of the kinematics and is due to a combination of time-dilation and frame transformation. The cross section for ground-state capture, without change of $\operatorname{spin}\left(\sigma_{\mathrm{ROBK} 1}^{\uparrow \uparrow}\right)$ then takes the simple form, to leading order in $\alpha Z$ :

$$
\sigma_{\mathrm{ROBK} 1}^{\uparrow \uparrow}(\gamma) \approx \frac{2^{5} \pi Z_{P}^{5} Z_{T}^{5}}{5 \gamma c^{12}}
$$

in atomic units.

The difficulty with calculating electron capture using this model is that the result, though Lorentz invariant, is not gauge invariant due to the nonorthogonality of the intial and final states. Moiseiwitsch and Stockman (1980) investigated this problem using a modification of the ROBK1 originally due to Bates. This defines a correction due to orthogonalization given by: $T_{\text {DRB }}=T_{\text {ROBK1 }}-T_{\text {corr }}$, where,

$$
T_{\text {corr }}(\boldsymbol{\eta})=(2 \pi)^{3} \int d^{3} \boldsymbol{s} \tilde{\phi}_{P}^{\dagger \prime}\left(\boldsymbol{K}^{\prime}+\boldsymbol{s}\right) S^{-1} \tilde{F}(\overline{\boldsymbol{s}}) S^{2} \tilde{V}_{P}^{\prime}(\boldsymbol{s}) \tilde{\phi}_{T}(\boldsymbol{K}+\overline{\boldsymbol{s}}) .
$$

In this expression

$$
\tilde{F}(\overline{\boldsymbol{s}})=(2 \pi)^{-3 / 2} \int d^{3} \boldsymbol{r} \mathrm{e}^{\mathbf{1} \overline{\boldsymbol{s}} \cdot \boldsymbol{r}} \phi_{T}^{\dagger}(\boldsymbol{r}) \phi_{T}(\boldsymbol{r}) .
$$

We use the overline symbol to denote a stretched vector thus: $\overline{\boldsymbol{s}}=\boldsymbol{s}_{\perp}+$ $\gamma s_{z} \hat{\boldsymbol{v}}$. In physical terms this correction term allows for capture through elastic scattering followed by the sudden adjustment of the wavefunction from target to projectile. Although this represents a two-step process, the matrix element is of first order in the interaction potential. In mathematical terms the new feature introduced in equation (7) is given by $\tilde{F}(\overline{\boldsymbol{s}})$ which is strongly peaked around $\overline{\boldsymbol{s}}=0$ and contains $\gamma$-dependent terms. The integral can be evaluated by the peaking approximation. One can show (Glass 1994) that the expression (17) is strongly determined by this peak, and to a high degree of accuracy one can simply replace the momentum distributions by their values at this peak: $\tilde{\phi}_{P}^{\dagger \prime}\left(\boldsymbol{K}^{\prime}+\boldsymbol{s}\right) \approx \tilde{\phi}_{P}^{\dagger \prime}\left(\boldsymbol{K}^{\prime}\right), \tilde{\phi}_{T}(\boldsymbol{K}+\overline{\boldsymbol{s}}) \approx \tilde{\phi}_{T}(\boldsymbol{K})$. Therefore the contribution of this term has a weight determined by the tails of the momentum distributions of the atoms, and as a result will be of higher order in $\alpha Z$ than the term $T_{\text {ROBK1 }}$. After making these assumptions, the 
integral poses no further difficulty and we obtain the following result for capture without spin flip:

$$
T_{\text {corr }}(\boldsymbol{\eta}) \approx-\frac{2^{5} \pi\left(Z_{P} Z_{T}\right)^{7 / 2}\left(\frac{\gamma+1}{2}\right)^{1 / 2} \ln [\gamma(1+v / c)]}{(v / c)\left(K^{2}+Z_{T}^{2}\right)^{2}\left(K^{\prime 2}+Z_{P}^{2}\right)^{2}}\left(1+\frac{K^{2}}{2 c^{2}}\right)
$$

The $(\ln \gamma)$ term is a consequence of the the binary scattering peak $\tilde{V}_{P}^{\prime}(\boldsymbol{s})$ and the presence of this factor will ultimately dominate the energy dependence so that as $\gamma \rightarrow \infty$ :

$$
\sigma_{\mathrm{DRB}}^{\uparrow \uparrow}(\gamma) \approx\left(\frac{32 \times 151}{21 \times 5}\right) \frac{\pi Z_{P}^{7} Z_{T}^{7}}{\gamma c^{16}}(\ln \gamma)^{2}
$$

in agreement with Moiseiwitsch and Stockman (1980) for capture from T(1s) to $\mathrm{P}(1 \mathrm{~s})$ without change of spin, and to leading order in $\alpha Z_{P, T}$. As mentioned by these authors, such a term requires extremely high energies and/or large charges for its presence to be significant.

\section{Second-order corrections}

The second-order term of the ROBK perturbation series gives the result (Glass et al 1996):

$$
\begin{array}{r}
T_{2}(\boldsymbol{\eta})=\int d^{3} \boldsymbol{s} \int d^{3} \boldsymbol{l} \tilde{\phi}_{P}^{\dagger \prime}\left(\boldsymbol{K}^{\prime}+\boldsymbol{l}\right) \tilde{V}_{P}^{\prime}(\boldsymbol{l}) S^{-1} \\
\times\left[\frac{c \boldsymbol{\alpha} \cdot(\boldsymbol{K}+\overline{\boldsymbol{l}}+\boldsymbol{s})+\beta c^{2}+E_{T}+\gamma \boldsymbol{v} \cdot \boldsymbol{l}}{\left(E_{T}+\gamma \boldsymbol{v} \cdot \boldsymbol{l}\right)^{2}-c^{2}(\boldsymbol{K}+\overline{\boldsymbol{l}}+\boldsymbol{s})^{2}-c^{4}+1 \epsilon}\right] S^{2} \tilde{V}_{T}(\boldsymbol{s}) \tilde{\phi}_{T}(\boldsymbol{K}+\boldsymbol{s}) .
\end{array}
$$

defined in the limit $\epsilon \rightarrow 0+$. The Dirac matrices are denoted by $\boldsymbol{\alpha}$ and $\beta$. If a direct comparison is to be made with experimental data this $T$-matrix should be calculated without further approximations. However, in order to make analytic progress, and if we are primarily concerned with the asymptotic form of the cross section, we can take $\alpha Z$ to be a good perturbative parameter, and seek the leading-order term of this integral. Historically, simplifying assumptions have been used in order to reduce this integral to a simple analytic form (Bransden and McDowell 1993). In general these methods relied upon peaking approximations which are known to have limited scope. 
As in section 1 the basic idea is that if an integrand possesses sharp and isolated peaks then the integral may be simplified. In expression (11) there are four significant peaks in the integrand at the values: $\boldsymbol{l}+\boldsymbol{K}^{\prime}=0, \boldsymbol{s}+\boldsymbol{K}=0$, and $\boldsymbol{l}=0, \boldsymbol{s}=0$. The first pair corresponds to bound-state momentum distributions while the second pair is associated with binary scattering. The fact that the peaks are sharp allows one to stretch the integration limits from the neighbourhood of the peak to infinity. And the isolation of the peaks permits one to separate each term. The leading term arises from the double bound-state peaks $\left(\boldsymbol{l}+\boldsymbol{K}^{\prime}=0, \boldsymbol{s}+\boldsymbol{K}=0\right)$. However there will be cross terms from the binary and bound-state peaks at ( $\boldsymbol{l}=0, \boldsymbol{s}+\boldsymbol{K} \equiv \boldsymbol{t}=0$ ) and at $\left(\boldsymbol{l}+\boldsymbol{K}^{\prime} \equiv \boldsymbol{T}=0, \boldsymbol{s}=0\right)$. Although these terms are are of higher order in powers of $\alpha Z$, we find that that they have a slower decay as a function of increasing energy and therefore dominate in the ultrarelativistic limit. Finally there will be a term of still higher order in $\alpha Z$ that arises from the double binary peak $(\boldsymbol{l}=0, \boldsymbol{s}=0)$. On the understandng that the peaks are sharp and isolated, then the expresssion (11) can be divided into four components as follows:

$$
T_{2}=T_{\mathrm{HM}}+T_{\mathrm{BPA}}+T_{\mathrm{BPB}}+T_{\mathrm{DBP}}
$$

The first term $\left(T_{\mathrm{HM}}\right)$ was evaluated by Humphries and Moiseiwitsch (1984). Retaining zeroth and first-order terms in $\boldsymbol{l}+\boldsymbol{K}^{\prime}$ and $\boldsymbol{s}+\boldsymbol{K}$ in the propagator, they obtained the expression:

$T_{\mathrm{HM}}(\boldsymbol{\eta}) \approx \frac{2^{4} \pi\left(Z_{P} Z_{T}\right)^{5 / 2}}{K^{2} K^{\prime 2}} \frac{\boldsymbol{\sigma}_{P}^{\dagger}(-\overline{\boldsymbol{K}}) S^{-1}\left(-c \boldsymbol{\alpha} \cdot \overline{\boldsymbol{K}}^{\prime}+\beta c^{2}+\gamma \epsilon_{f}\right) S^{2} \boldsymbol{\sigma}_{T}\left(\overline{\boldsymbol{K}}^{\prime}\right)}{\left(2 \gamma E_{T} E_{P}-c^{4}-\epsilon_{f}^{2}-K^{2} c^{2}+2 i Z_{P} \bar{K} c^{2}+2 i Z_{T} \bar{K}^{\prime} c^{2}\right)}$

where $\boldsymbol{\sigma}(\boldsymbol{k})$ denotes a Darwin spinor

$$
\boldsymbol{\sigma}_{T, P}(\boldsymbol{k})=\left(1+\frac{i \boldsymbol{\alpha} \cdot \boldsymbol{k} Z_{T, P}}{2 k c}\right) w_{T, P}
$$

In this notation the spin vector is defined as $\tilde{w}_{P, T}=\left(\begin{array}{llll}1 & 0 & 0 & 0\end{array}\right)$ for spin up. For $\alpha Z_{P, T} \ll 1$ we get

$$
T_{H M}(\boldsymbol{\eta})=\frac{2^{5} \pi\left(Z_{P} Z_{T}\right)^{5 / 2}\left(\frac{\gamma+1}{2}\right)^{1 / 2} F}{K^{2} K^{\prime 2}\left(2 c^{2}(\gamma-1)-K^{2}+2 i Z_{P} \bar{K}+2 i Z_{T} \bar{K}^{\prime}\right)}
$$


where $F=\gamma+\frac{1}{2}\left(\frac{\gamma-1}{\gamma+1}\right)$ for nonflip ( $\left.\uparrow\right)$ and $F=x(2 \gamma+1) \eta / 2 c$ for capture with spin flip $(\uparrow \downarrow)$.

This approximate form for the ROBK2 transition amplitude has to be qualified on several counts. Firstly, we would expect the peaking approximations used in this derivation to break down for large charges and/or low velocities; neither criterion is strongly satisfied for much of the experimental data (Anholt 1985). However, it transpires that total cross sections within this approximation are in considerably better agreement with experiment than the ROBK1 results, which are commonly five times too large. On average the Humphries and Moiseiwitsch (HM) approximation to ROBK2 is around a factor of two lower than ROBK1. This still leaves a considerable gap between theory and experiment. Moiseiwitsch (1988) introduced the 'averaging approximation' in which the external $K^{\prime 2} K^{2}$ factors in the denominator of equation (15) are replaced by $K^{\prime 2}+Z_{P}^{2}$ and $K^{2}+Z_{T}^{2}$, which resulted in a dramatic improvement between theory and experiment.

In 1990 Decker calculated ROBK2 without approximation and using fully relativistic wavefunctions. It was discovered that in many cases rather than improving on the ROBK1 predictions, exact ROBK2 was an order of magnitude greater than ROBK1 (Eichler 1990), and consequently in poorer accord with experiment. This obviously raises serious doubts concerning the validity of the various peaking approximations discussed above, especially for large charges and energies in the lower range of the relativistic regime. There has been considerable debate in the literature as to which method is correct, with Decker and Eichler (1993) arguing in favour of exact numerical calculation of the perturbation series, while Moiseiwitsch (1995) has pointed out the possible pitfalls of simultaneously expanding along the Born series and in terms of $\alpha Z$ in the wavefunctions.

\subsection{Binary scattering peaks}

In section 1 it was shown that the binary scattering peak can produce logarithmic terms though with a small coefficent. The contribution of such terms within second-order perturbation theory can be evaluated without much difficulty so long as the assumption that the peaks are sharp and isolated holds true. This supposes, for example, that the peaks at $\boldsymbol{l}=\mathbf{0}$ and $\boldsymbol{l}=-\boldsymbol{K}^{\prime}$ are distinct, which is certainly the case so long as $\alpha Z_{P} \ll 1$. In this calculation we continue to make this assumption, using $\alpha Z$ as a perturbation parameter, 
in order to obtain the leading-order correction from the binary peaks.

The first binary peak of (12) leads to the term:

$$
\begin{array}{r}
T_{\mathrm{BPA}}(\boldsymbol{\eta}) \approx \int d^{3} \boldsymbol{t} \int d^{3} \boldsymbol{l} \tilde{\phi}_{P}^{\dagger \prime}\left(\boldsymbol{K}^{\prime}+\boldsymbol{l}\right) \tilde{V}_{P}^{\prime}(\boldsymbol{l}) S^{-1} \\
\times\left[\frac{c \boldsymbol{\alpha} \cdot \overline{\boldsymbol{l}}+\beta c^{2}+E_{T}+\gamma \boldsymbol{v} \cdot \boldsymbol{l}}{E_{T}^{2}-c^{4}+2 \gamma E_{T} \boldsymbol{v} \cdot \boldsymbol{l}-2 c^{2} \boldsymbol{t} \cdot \overline{\boldsymbol{l}}-c^{2} l^{2}+1 \epsilon}\right] \times S^{2} \tilde{V}_{T}(-\boldsymbol{K}) \tilde{\phi}_{T}(\boldsymbol{t}) .
\end{array}
$$

and a similar term will aries from the second peak at: $\boldsymbol{l}+\boldsymbol{K}^{\prime} \equiv \boldsymbol{T}=0, \boldsymbol{s}=0$.

In the usual manner, we can invert the Fourier transform for $\tilde{\phi}_{T}(\boldsymbol{t})$ by using the identity

$$
(\lambda+1 \epsilon)^{-1}=-i \int_{0}^{\infty} d u \exp (i u(\lambda+i \epsilon))
$$

to convert the denominator into an exponential. Explicitly, we have for 1sstates the expression:

$$
\begin{aligned}
& T_{\mathrm{BPA}}(\boldsymbol{\eta}) \approx(2 \pi)^{3 / 2}\left(Z_{T}^{3} / \pi\right)^{1 / 2} \tilde{\phi}_{P}^{\dagger \prime}\left(\boldsymbol{K}^{\prime}\right) \tilde{V}_{T}(-\boldsymbol{K}) \int d^{3} \boldsymbol{l} \tilde{V}_{P}^{\prime}(\boldsymbol{l}) S^{-1} \\
& \quad \times\left[\frac{c \boldsymbol{\alpha} \cdot \overline{\boldsymbol{l}}+\beta c^{2}+E_{T}+\gamma \boldsymbol{v} \cdot \boldsymbol{l}}{E_{T}^{2}-c^{4}+2 \gamma E_{T} \boldsymbol{v} \cdot \boldsymbol{l}-c^{2} l^{2}+2 i c^{2} Z_{T} \bar{l}+i \epsilon}\right] S^{2} \boldsymbol{\sigma}_{T}(-\overline{\boldsymbol{l}})
\end{aligned}
$$

To leading order in $\alpha Z$ we have

$$
T_{\mathrm{BPA}}(\boldsymbol{\eta})=(2 \pi)^{3 / 2}\left(Z_{T}^{3} / \pi\right)^{1 / 2} \tilde{\phi}_{P}^{\dagger \prime}\left(\boldsymbol{K}^{\prime}\right) \tilde{V}_{T}(-\boldsymbol{K}) S^{-1}(\beta+1) S^{2} w_{T} L
$$

with

$$
L=-\int d^{3} \boldsymbol{l} \tilde{V}_{P}^{\prime}(\boldsymbol{l}) \frac{1}{\left(l^{2}-2 \boldsymbol{v} \cdot \overline{\boldsymbol{l}}+Z_{T}^{2}-2 i Z_{T} \bar{l}-i \epsilon\right)}
$$

Even after these simplifications, the calculation of this integral is not straightforward. However, it is clear that the main feature of the integrand is due to the singularity in the propagator. It is this factor rather than the potential scattering in (20) that gives rise to the logarithmic behaviour. An analytic form can be found for this integral (Glass 1994) though its form is rather complicated. Instead it can be shown to a fair approximation that this complicated form reduces to,

$$
L \approx-i(2 \pi)^{3 / 2}\left(Z_{P} / \gamma v\right) \ln \left(2 i \gamma Z_{T} / v\right)
$$


Then within these approximations we have a simple formula for groundstate to ground-state capture without change of spin:

$$
T_{\mathrm{BPA}}^{\uparrow \uparrow} \approx-\left(2 i Z_{P} / v\right) \ln \left(2 i \gamma v / Z_{T}\right) T_{\mathrm{ROBK} 1}^{\uparrow \uparrow}
$$

Using the same method we find that the term due to the peaks around $\boldsymbol{l}+\boldsymbol{K}^{\prime} \equiv \boldsymbol{T}=0, \boldsymbol{s}=0$. is approximately given by:

$$
T_{\mathrm{BPB}}^{\uparrow \uparrow} \approx-\left(2 i Z_{T} / v\right) \ln \left(2 i \gamma v / Z_{P}\right) T_{\mathrm{ROBK} 1}^{\uparrow \uparrow}
$$

While these terms are of higher order than ROBK1 in $\alpha Z$, they are are of lower order than the Bates term given by equation (9). Furthermore it is clear that in the limit of extreme relativistic energies that this term decreases much more slowly than the bound-state peaking term (15). If we combine these terms according to (12) the result is that at relativistic energies the nonradiative electron capture cross section has the asymptotic form:

$$
\sigma_{\mathrm{ROBK} 2}^{\uparrow \uparrow}(\gamma) \sim \frac{2^{7} \pi Z_{T}^{5} Z_{P}^{5}\left(Z_{T}+Z_{P}\right)^{2}}{5 c^{14}} \frac{(\ln \gamma)^{2}}{\gamma}
$$

It is clear from this expression that the ROBK2 approximation contains logarithmic terms and that these are in general more important than the term from the Bates approximation (10) for small $\alpha Z$. The simple reason is that only one momentum tail is sampled in producing the term (19). To complete our discussion, we note that additional logarithmic terms will be produced by the product of the two binary peaks: term $T_{\mathrm{DBP}}$ in equation (12). However, these will be of higher order in $\alpha Z$ than the term (19) because they include terms from both momentum tails as in the Bates approximation. Hence their contribution will not be a significant factor in this process for $\alpha Z \ll$ 1. Finally we conclude by mentioning that capture with spin-flip through this process contributes a small additional term. Further consideration of equation (19) for the case of capture with spin-flip shows that, in common with ROBK1, the cross section is only a quarter of the non-flip result ( $\sigma^{\uparrow \downarrow}=$ $\frac{1}{4} \sigma^{\uparrow \uparrow}$ ). Previously, calculations using ROBK2 (with bound-state peaking) and the RCDW approximation (Glass et al 1994), showed that for relativistic energies capture with spin flip is more probable than capture without spin flip. From the analysis above, we see that this is not the case in the extreme relativistic regime. 


\section{Conclusions}

In the Bates approximation logarithmic effects arise from scattering at the on-shell binary peaks. Our estimate of the corresponding terms in secondorder perturbation theory confirm that there are indeed logarithmic terms, and the magnitude of these terms is larger than those obtained from the nonorthogonal correction introduced by Moiseiwitsch and Stockman (1980). This supports the claims made by Decker (1990) and Jakubassa-Amundsen and Amundsen (1985) in highlighting the cause of this effect and, more importantly estimates the coefficient of the logarithmic term. The total cross section including transitions involving spin flip is given by the formula:

$$
\sigma_{\mathrm{ROBK} 2}(\gamma) \sim 2^{5} \pi \alpha^{14} Z_{T}^{5} Z_{P}^{5}\left(Z_{T}+Z_{P}\right)^{2} \gamma^{-1}(\ln \gamma)^{2}
$$

Interestingly, the logarithmic correction is not principally the result of retardation as previously supposed (Jakubassa-Amundsen and Amundsen 1985) but rather due to propagator singularities. In other words, it is a principally a kinematic feature. Furthermore the correction will only be significant for extreme energies and thus is primarily of academic interest. Lastly we note

that capture without change of spin is the dominant transition at extremely high energies.

\section{References}

Anholt R 1985 Phys. Rev. A 313579.

Bransden B H and McDowell M R C 1993 Charge Exchange and the Theory of Ion-Atom Collisions (Oxford: Clarendon Press).

Decker F 1990 Phys. Rev. A 416552.

Decker F and Eichler J 1993 J. Phys. B: At. Mol. Opt. Phys. 262081.

Deco G R and Rivarola R D 1987 J.Phys.B:At.Mol.Opt.Phys. 203853.

Eichler J 1990 Phys. Rep. 193165.

Eichler J and Meyerhof W E 1995 Relativistic Atomic Collisions (New York: Academic Press). 
Glass J T, McCann J F and Crothers D S F 1994 J.Phys.B:At.Mol.Opt.Phys. 273445.

—1996 Z.Phys. D 36119.

Glass J T 1994 Ph. D. Thesis, Queen's University Belfast, unpublished.

Humphries W J and Moiseiwitsch B L 1984 J.Phys.B:At.Mol.Phys. 17 2655.

Jakubassa-Amundsen D H and Amundsen P A 1980 Z. Phys. A 29813.

—1985 Phys. Rev. A 323106.

Moiseiwitsch B L 1986 J.Phys.B:At.Mol.Phys. 193733.

—1988 J.Phys.B:At.Mol.Phys. 21603.

-1995 XIX Int. Conf. Physics Elec. At. Collisions (Whistler, Canada) Book of Abstracts, eds. Mitchell J B A et al p 107.

Moiseiwitsch B L and Stockman S G 1979 J.Phys.B:At.Mol.Phys. 12 L695.

Moiseiwitsch B L and Stockman S G 1980 J.Phys.B:At.Mol.Phys. 134031.

Mukherjee S C, Sural D P, McCann J F and Shimamura I 1992 Comm. At. Mol. Phys. 2825.

Shakeshaft R 1979. Phys. Rev. A 20779.

Shakeshaft R and Spruch L 1979 Rev. Mod. Phys. 51369.

Toshima N and Eichler J 1990 Phys. Rev. A 40125. 